\title{
Asymmetric Turbo Code for Coded-Cooperative Wireless Communication Based on Matched Interleaver with Channel Estimation and Multi-Receive Antennas at the Destination
}

\author{
Shoaib MUGHAL, Fengfan YANG, Saqib EJAZ, Syed Hassan AHMED, Rahim UMAR \\ College of Electronic and Information Engg., Nanjing Univ. of Aeronautics and Astronautics, Nanjing 210016, China \\ mughalshoaib@hotmail.com,yffee@nuaa.edu.cn,saqib.ejaz@foxmail.com,syedhassan_ahmad@hotmail.com, \\ rahim_nuaa@hotmail.com
}

Submitted October 20, 2016 / Accepted June 3, 2017

\begin{abstract}
This paper investigates the multiple relay codedcooperation scheme based on asymmetric turbo code (ATC) with multiple receive antennas over Rayleigh block fading channels. An encoding scheme based on ATC is proposed for coded-cooperation, i.e. distributed asymmetric turbo code (DATC). The code matched interleaver (CMI) is selected by a rigorous comparison with a uniform-random interleaver (URI). This optimum choice of interleaver at the relay nodes provides maximum benefit from DATC coded-cooperation scheme. Practically in any wireless communication system, the channel side information (CSI) is usually unknown at the receiver. Therefore, spatial normalized least mean square (NLMS) adaptive transversal filters are employed to estimate the CSI at the destination node. Moreover, in coded-cooperation scheme, the effectiveness and validation of spatial NLMS adaptive transversal filters is also verified by simulation results. Quadrature phase shift keying (QPSK) is used in codedcooperation scheme and corresponding soft-demodulators are employed along with joint iterative soft-input soft-output (SISO) decoder at the destination node. Monte Carlo simulations show that the proposed scheme incorporates coding gain, diversity gain and cooperation gain successfully, which eventually results in net gain of 2.7 to $3.5 \mathrm{dBs}$ over non-cooperation ATC counterpart.
\end{abstract}

\section{Keywords}

Joint iterative SISO decoder, coded-cooperation, distributed asymmetric turbo code, CMI, multipleinput multiple-output (MIMO), channel side information

\section{Introduction}

Recently, there has been a rapid increase in the demand of high speed data rates in wireless communication. Fading is one of the main adverse factors that affect the speed of high data rate in wireless communication. This problem of fading can be solved by employing diversity techniques such as time, frequency, and spatial diversities [1]. Spatial diversity is one of the key diversity techniques extensively used in literature [1], [2] to mitigate the harsh effects of fading channels, thereby enhancing the performance of wireless communication system. The simplest way to get the spatial diversity is to employ multiple antennas both at transmitter and receiver ends [1], [2]. However, in typical mobile equipment, there has been an always inevitable constraint due to limitations in weight, size and area etc. Thus, deploying multiple antennas in mobile equipment is not a feasible option in today's present world. The problem in this situation can be compensated by using three-terminal or relay assisted cooperative system commonly known as virtual MIMO [3]. In a virtual MIMO, each of the mobile equipment contains a single antenna, forms a group and communicates with each other in a cooperative manner. Thus, it takes the benefits of MIMO technique by creating a virtual antenna array.

The idea of relay assisted cooperative system was an outcome of the pioneered efforts made by Van der Meulen [4] to overcome the existing problem of single antenna deployment in a typical mobile device. Relay assisted cooperative communication is fundamentally based on communication protocols such as amplify-and-forward (AF) [5], compress-and-forward (CF) [6] and decode-andforward (DF) [7]. The performance efficient user cooperation based on ad-hoc wireless commination is detailed in [5]. The implementation issues of user cooperation for wireless networks are discussed in [8]. Recently, there has been an evolution in the ground breaking idea of relay assisted cooperative system called coded-cooperation. The coded-cooperation scheme [9] is a combination of cooperative scheme with existing channel coding like Turbo codes [10], Low Density Parity Check (LDPC) codes [11], Convolution codes [12], Reed-Muller code [13] and Polar codes $[14,15]$. The basic idea of coded-cooperation is to distribute the channel codes over source and relay terminals, i.e. overall code-word reaches the destination by independent fading paths. Thus, the information bits are 
recovered by using joint decoding scheme [16] deployed at the destination node. Recently, the author in [16] described the concept of coded-cooperation in detail by considering turbo code as distributive channel code.

In literature, coded-cooperative schemes have been mostly developed for Binary Phase Shift Keying (BPSK) modulation scheme but these schemes are bandwidth inefficient. However, bandwidth efficiency can be improved by using higher order modulation schemes like QPSK, 8-PSK, 16-PSK or other higher order modulation schemes [17]. Thus, QPSK has been considered as a modulation scheme in this paper. Moreover, we have considered ATC in coded-cooperation as to the best of our knowledge all the previous research has been done on Symmetric Turbo codes (STC) in coded-cooperation. The proposed scheme is capable of accomplishing coding gain and diversity gain with increased bandwidth efficiency. Diversity gain is achieved from multi-receive antennas while transmission links from source to destination and relays to destination provide cooperation diversity.

Turbo code performance is based on distance spectrum of the code [18]. Distance spectrum of the code can be improved by using component encoder with large minimum free distance $d_{\text {free. }}$ Interleaver design is a crucial part of turbo code which plays an important role in improving the effective free distance of the code. The basic purpose of interleaver is to remove the bad weight code-words. The bad weight code-words are usually produced by low weight information sequences such as weight-2, 3 and 4. Different interleavers have been reported in literature [19-22]. For ATC, it has been suggested in the literature [23] that CMI outperforms uniform-random interleaver (URI) in additive white Gaussian noise (AWGN) and Rayleigh block fading channels for non-cooperative communication. In order to enhance the error performance of coded-cooperative communication system, CMI is selected as an optimum choice of interleaver in this paper. CSI is usually not known to the receiver so CSI estimation is required. Therefore, we propose a training sequence based spatial normalized least mean square (NLMS) adaptive transversal algorithm for the channel estimation at the destination [24-26]. The performance of DATC for CMI with multi-receive antenna with perfect and imperfect CSI have been analyzed.

The major contributions of this manuscript can be summarized as below:

- The ATC is used for the first time in multi-relay coded cooperative communication to the best of our knowledge.

- The ATC with CMI is used in multi-relay coded cooperative communication.

- The channel estimation is performed by spatial NLMS adaptive filter in coded cooperative communication.

The remaining manuscript is organized as follows. Section 2 presents the fundamentals of general coded-cooperative scheme with multiple relays, and multiple receive antennas at the destination node. Section 3 mainly deals with the fundamentals of ATCs and describes the proposed multi-relay coded-cooperative scheme. Moreover, it discusses the CMI design for ATC schemes. Section 4 proposes a spatial NLMS adaptive filter for channel estimation. Soft demodulation and joint iterative SISO decoder for DATC scheme are explained in Sec. 5 and Sec. 6, respectively. In Sec. 7, simulation results of coded-cooperative schemes for ATC in different scenarios are presented and discussed. Finally, the paper is concluded with the conclusion drawn in Sec. 8.

\section{Model of Coded-Cooperation Communication with Multi-Relays and Multi-Receive Antennas}

A basic half-duplex coded-cooperation model with multi-relays and multi-receive antennas is shown in Fig. 1. The source $S$ node and relay $\left(R_{\omega}, \omega=1\right.$ to $\left.L_{\mathrm{R}}\right)$ nodes are composed of single antenna as they are considered to be user equipment (UE), where $L_{\mathrm{R}}$ is representing the total number of relay nodes. The destination node $D$ can be viewed as a base station as it contains multiple receive antennas. One complete transmission from source to destination node requires two time slots. During the first time slot, the source node broadcasts information sequence to relay nodes and a destination node. During the second time slot, all relay nodes transmit their information sequence to the destination node. Moreover, the relay nodes are placed far apart from each other in such a way that they transmit their corresponding information sequences over non overlapping channels.

At the source node, code $C_{1}$ encodes the binary sequence of bits $\mathbf{u}_{1}$ to code-word $\mathbf{c}_{\mathrm{S}}$. The modulation is performed on codeword $\mathbf{c}_{\mathrm{S}}$ to get the modulated sequence of symbols which is denoted by $\mathbf{x}_{\mathrm{S}}=\left[x_{\mathrm{S}}^{1}, x_{\mathrm{S}}^{2}, \ldots, x_{\mathrm{S}}^{N_{1}^{\mathrm{s}}}\right]$, where $x_{\mathrm{S}}^{j}\left(j=1,2, \ldots, N_{1}^{\mathrm{S}}\right)$ is the modulated symbol and $N_{1}^{\mathrm{S}}$ is the length of this sequence. Modulated sequence of symbols is broadcasted to the relay nodes and destination node. During the first time slot, the received coded symbol vector $\mathbf{r}_{\mathrm{SD} \varrho}$ at the destination's $\varrho$ th $(\varrho=1$ to $L)$ antenna is modelled as follows

$$
\mathbf{r}_{\mathrm{S} D_{\varrho}}=\mathbf{h}_{\mathrm{S} D_{\varrho}} \mathbf{x}_{\mathrm{S}}+\mathbf{n}_{\mathrm{S} D_{\varrho}}
$$

where $\mathbf{h}_{\mathrm{S} D_{\rho}}=\left[h_{\mathrm{S} D_{\rho}}(1), h_{\mathrm{S} D_{\rho}}(2), \ldots, h_{\mathrm{S} D_{\rho}}\left(N_{1}^{\mathrm{s}}\right)\right]$ is a Rayleigh fading channel vector, $h_{\mathrm{S} D_{\rho}}(j) \sim R(0,1),\left(j=1,2, \ldots, N_{1}^{\mathrm{s}}\right)$

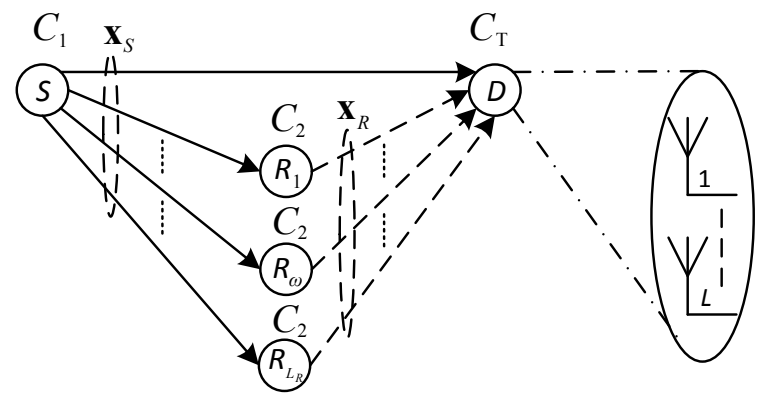

Fig. 1. Multi-relay coded-cooperation communication model. 
is zero mean and unit variance complex Gaussian random variable. Since Rayleigh block fading is assumed therefore $\mathbf{h}_{\mathrm{S} D \varrho}$ becomes $\mathbf{h}_{\mathrm{S} D_{\varrho}}=\left[h_{\mathrm{S}_{\varrho}}(1)=h_{\mathrm{S} D_{\varrho}}(2)=, \ldots,=h_{\mathrm{S} D_{\varrho}}\left(N_{1}^{\mathrm{s}}\right)\right]$. The vector $\quad \mathbf{n}_{\mathrm{S}_{D_{\varrho}}}=\left[n_{\mathrm{S} D_{\varrho}}(1), n_{\mathrm{S} D_{\varrho}}(2), \ldots, n_{\mathrm{SD}_{\varrho}}\left(N_{1}^{\mathrm{s}}\right)\right]$ is an AWGN, $n_{\mathrm{S} D_{e}}(j) \sim N\left(0, \sigma^{2}\right),\left(j=1,2, \ldots, N_{1}^{\mathrm{s}}\right)$ is zero mean and $\sigma^{2} / 2$ variance per dimension. The sequences received at each antenna at the destination node are joined using maximum ratio combining (MRC) technique to get $\mathbf{r}_{\mathrm{SD}}$ sequence.

The $\omega$-th relay node receives the coded symbol vector $\mathbf{r}_{\mathrm{S} R_{\omega}}$ during the first time slot that is modelled as below

$$
\mathbf{r}_{\mathrm{S} R_{\omega}}=\mathbf{h}_{\mathrm{S} R_{\omega}} \mathbf{x}_{S}+\mathbf{n}_{\mathrm{S} R_{\omega}}
$$

where $\mathbf{h}_{\mathrm{S} R_{\omega}}$ is a Rayleigh fading channel vector and $\mathbf{n}_{\mathrm{S} R_{\omega}}$ is a vector of AWGN and their definitions are similar as $\mathbf{h}_{\mathrm{S} D_{\varrho}}$ and $\mathbf{n}_{\mathrm{S} D_{\varrho}}$, respectively. The detection process at $\omega$-th relay node is divided into two steps. The first step consists of soft demodulation of received coded symbol vector $\mathbf{r}_{\mathrm{S} R_{\omega}}$ into soft coded bits. In the second step, SISO decoder decodes soft coded bits. The source to $\omega$-th relay $\left(S-R_{\omega}\right)$ channel is usually considered as ideal $\left(\mathrm{SNR}_{\mathrm{S} R_{\omega}}=\infty\right)$ to have a perfect decoding at relay node. In case of non-ideal $\left(\mathrm{SNR}_{\mathrm{SR}_{\omega}} \neq \infty\right) S-R_{\omega}$ channel, different cooperation protocols have been proposed in literature. Cyclic redundancy check (CRC) [9] is mostly applied to ensure error free decoding at the relay node. In this paper, ideal $S-R_{\omega}$ channel is assumed for proposed coded-cooperation schemes.

At the $\omega$-th relay node, code $C_{2}$ encodes the decoded bits again. These encoded bits are transmitted to the destination node after modulation in the second time slot. The modulated sequence of symbols is denoted by $\mathbf{x}_{R}=\left[x_{R}^{1}, x_{R}^{2}, \ldots, x_{R}^{N_{1}^{R}}\right]$ where $x_{R}^{j},\left(j=1,2, \ldots N_{1}^{R}\right)$ is the modulated symbol and $N_{1}^{R}$ is the length of this sequence. The received sequence at the destination's $\varrho$-th antenna during second time slot is modelled as

$$
\mathbf{r}_{R D_{\varrho}}=\mathbf{h}_{R D_{\varrho}} \mathbf{x}_{R}+\mathbf{n}_{R D_{\varrho}}
$$

where $\mathbf{h}_{\mathrm{R} D_{\varrho}}$ is a Rayleigh fading channel vector and $\mathbf{n}_{\mathrm{R} D_{\varrho}}$ is a vector of $A W G N$ and their definition are similar as $\mathbf{h}_{\mathrm{S} D_{\varrho}}$ and $\mathbf{n}_{\mathrm{S} D_{\varrho}}$, respectively.

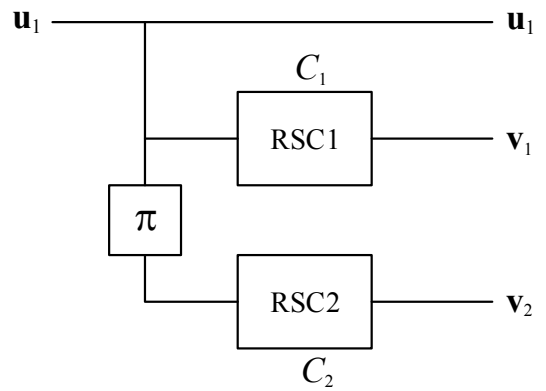

Fig. 2. Asymmetric turbo code.
At the destination node, the antenna array receives the signal that was transmitted by the relay node. The destination node combines them by using MRC technique to get the $\mathbf{r}_{R D}$ sequence. The channels are assumed to be Rayleigh block fading channels. Moreover, the channels remain static for a complete code-word. Furthermore, the transmission power at each node is assumed to be equal.

\section{ATC and DATC with Code Matched Interleaver}

This section discusses the basic structure of ATC. The two different RSCs in ATC are distributed in such a way that one RSC is placed at source node while other RSC is deployed at the relay node. Thus, this parallel encoding structure of ATC makes it suitable for coded-cooperation therefore a DATC scheme with CMI is discussed.

\subsection{Asymmetric Turbo Code (ATC)}

The turbo code is mostly a parallel concatenation of two RSC encoders with an interleaver $\pi$ connected in a manner as shown in Fig. 2. Unlike conventional STC, ATC is a combination of two different constituent RSC encoders and decoders.

A parallel concatenated turbo code is denoted by $C_{\mathrm{T}}=\left[C_{1}, C_{2}\right]$, where $C_{1}$ and $C_{2}$ are RSC codes with only the trellis of $C_{1}$ is terminated. For conventional STC $C_{1}$ and $C_{2}$ codes are identical but for ATC $C_{1}$ and $C_{2}$ codes are different. An RSC is denoted by generator matrix $\mathbf{G}\left(\mathrm{g}_{\mathrm{b}}(D) / \mathrm{g}_{\mathrm{a}}(D)\right)$ where $\mathrm{g}_{\mathrm{a}}(D)$ is feedback polynomial while $\mathrm{g}_{\mathrm{b}}(D)$ is feed-forward polynomial in octal form. The turbo code with primitive feedback polynomial in component codes is called as primitive turbo code. The turbo codes discovered by Berrou et al. [27] are an example of nonprimitive turbo codes.

The BER performance of turbo code is basically evaluated on two distinct regions, namely error-floor and waterfall region. The conventional STC either performs well in the error-floor region or in the waterfall region but not in both regions simultaneously [28]. The non-primitive STC performs well in the waterfall region while the primitive STC shows good performance in the error-floor region.

The performance of a turbo code is based on various parameters such as block length, component codes, interleaver design and the distance spectrum properties. The ATC has a capability to provide good distance spectrum properties that enables it to perform well in both regions, i.e. waterfall and error-floor [23]. At the same time choosing suitable component codes depends upon the generator matrix as it plays a vital role in determining the overall weight of code-words [23].

In this paper, ATC code having different RSCs for constraint length $K_{L}=4$ is being considered. The ATC with generator matrix $\mathbf{G}(13 / 15,11 / 15)_{8}$ is selected based on its better BER performance [23]. This ATC is used in the proposed coded-cooperation scheme. 


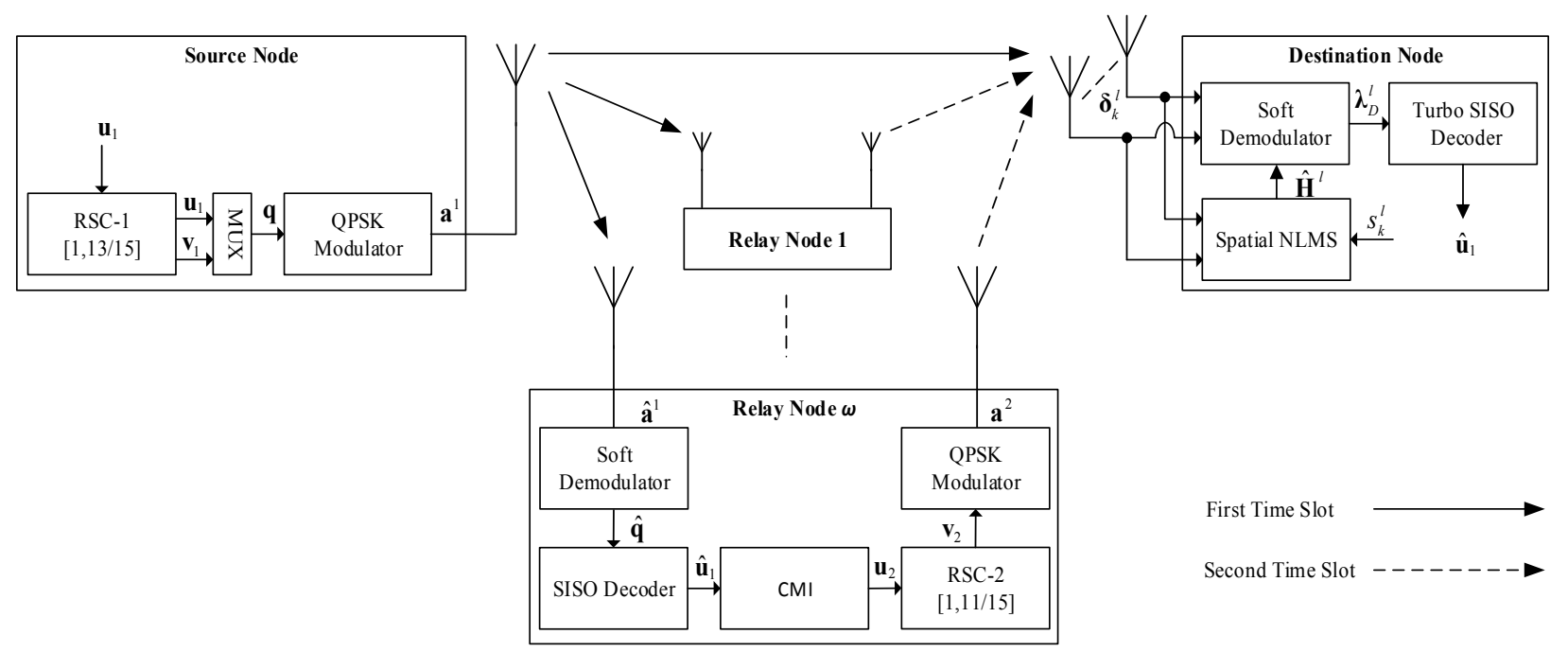

Fig. 3. Distributed multi-relay asymmetric turbo code.

\subsection{Distributed Asymmetric Turbo Code (DATC)}

The structure of turbo code is perfectly suitable to be effectively used in coded-cooperative scenarios. Turbo codes which are formed as the result of cooperation between source and relay nodes are usually called as Distributed Turbo Codes (DTC) in literature [29]. If the RSCs at the source and relay nodes are different then this DTC is termed as DATC. The generator polynomial use for our proposed DATC scheme is $\mathbf{G}(13 / 15,11 / 15)_{8}$. This scheme takes two time slots for transmission of a complete turbo code frame. The source node consists of $1 / 2$ rate $\mathrm{RSC}-1$ encoder and QPSK modulator. The source node converts information sequence $\mathbf{u}_{1}$ of length $K$ into coded sequence $\mathbf{q}$ of length $N_{1}$. These coded bits are mapped onto QPSK constellation $\mathcal{M}(\mathbf{q})$ to form coded symbol sequence $\mathbf{a}^{1}$ that contains $N_{1}{ }^{\mathrm{S}}$ symbols. The source node simultaneously broadcasts these $N_{1}^{\mathrm{S}}$ symbols $\mathbf{a}^{1}$ to all relay nodes and the destination node in the first time slot. The destination node consists of multi-receive antennas. The relay nodes are usually placed closer to the destination node in a typical coded-cooperation scenario [30]. Therefore, we also have placed all the relay nodes near to the destination node.

The $\omega$-th relay node demodulates $N_{1}^{\mathrm{S}}$ received coded symbol sequence $\hat{\mathbf{a}}^{\mathbf{1}}$ to $N_{1}$ soft information sequence $\hat{\mathbf{q}}$ and this soft information sequence is decoded by SISO decoder to get the estimated information sequence $\hat{\mathbf{u}}_{\mathbf{1}}$. If the estimated information sequence $\hat{\mathbf{u}}_{\mathbf{1}}$ is error free then it is called ideal coded-cooperation otherwise it is called non-ideal coded-cooperation. Estimated information sequence $\hat{\mathbf{u}}_{\mathbf{1}}$ is interleaved using CMI. The details of designing CMI are given in the subsequent subsection. This interleaved information sequence $\mathbf{u}_{2}=\pi\left(\hat{\mathbf{u}}_{1}\right)$ is given as an input to $1 / 2$ rate RSC-2 encoder at relay node. Output of RSC-2 encoder contains systematic and parity bit sequences. Systematic bit sequence of RSC-2 encoder is suppressed and parity bit sequence $\mathbf{v}_{2}$ of length $N_{2}$ is further passed through QPSK modulator. The modulated sequence $\mathbf{a}^{2}$ of length $N_{2}{ }^{\mathrm{R}}$ is send to the destination node in the second time slot. Simi- larly all the relay nodes transmit their modulated sequences in the second time slot.

The channels are estimated by spatial NLMS adaptive filters using training sequence for each time slot. With the help of estimated channels, the received symbol sequence from each receive antenna are converted into log likelihood ratios (LLRs). These LLRs are combined using maximum ratio combining (MRC) technique. Combined LLRs are given as input to soft demodulator that results in soft bit LLRs. These soft bit LLRs for the first and second time slots are given as input to the joint iterative SISO turbo decoder to estimate the information sequence. The whole process of joint iterative SISO decoding is explained in the subsequent section. Figure 3 shows the DATC scheme. The overall code rate for DATC is $R_{\mathrm{c}}=1 / 3$.

\subsection{Code Matched Interleaver (CMI)}

The performance of a code is decided by its distance properties. The distributive construction of a code with good distance spectrum plays a crucial role in coded-cooperation. The distance spectrum of turbo code depends on interleaver; therefore interleaver design is the vital part of turbo code.

CMI is a better variant of S-random interleaver [21]. It improves the distance spectrum of ATC by removing low-weight code-words which are usually generated by low-weight information sequences such as weight-2, 3 and 4. Although all the low weight (weight-2, 3 and 4) information sequences are not bad so CMI only considers those low weight information sequences which produces low weight code-words. The performance of turbo code is based on its free distance in high SNR regime but it is dependent on the size of interleaver in low to medium SNR regime [22].

CMI mainly improves the performance of turbo code by removing low-weight information sequences which generate the low-weight code-words [23]. The design steps of CMIs for STC and ATC are discussed in literature [22], 
[23]. However, we briefly discuss the construction of CMI for ATC which removes bad weight code-words and improves the performance of ATC.

Suppose that the RSC-1 encoder encodes $\mathbf{u}_{1}$ information sequence to $\left(\mathbf{u}_{1}, \mathbf{v}_{1}\right)$ sequence pair. Similarly RSC-2 encoder generates $\left(\mathbf{u}_{2}, \mathbf{v}_{2}\right)$ sequence pair after encoding the $\mathbf{u}_{2}$ information sequence where $\mathbf{u}_{2}$ is interleave form (URI) of $\mathbf{u}_{1}$ information sequence. $\mathbf{v}_{1}$ and $\mathbf{v}_{2}$ are parity sequences of RSC-1 and RSC-2, respectively. Assume that the weight of information sequences $\mathbf{u}_{1}$ and $\mathbf{u}_{2}$ is denoted by $\alpha$. The weight of parity sequences of RSC- 1 and RSC-2 are denoted by $\alpha\left(\mathbf{v}_{1}\right)$ and $\alpha\left(\mathbf{v}_{2}\right)$, respectively. The weight of an ATC code-word is given as follows

$$
d=\alpha+\alpha\left(\mathbf{v}_{1}\right)+\alpha\left(\mathbf{v}_{2}\right) .
$$

The polynomial representation of weight-2 information sequence $\mathbf{u}_{1}^{2}$ which results in finite weight codeword is given as

$$
\mathbf{u}_{1}^{2}(D)=\left(1+D^{\varphi_{1} k_{1}}\right) D^{\zeta_{1}}
$$

where $\varphi_{1}$ is the minimum spacing between pair of "1"s of weight-2 information sequence $\mathbf{u}_{1}^{2}, k_{1}=1,2,3,4, \ldots$ and $\zeta_{1}=1,2,3,4, \ldots$ is time delay. The polynomial representation of interleave information sequence $\mathbf{u}_{2}{ }^{2}$ is given as

$$
\mathbf{u}_{2}^{2}(D)=\left(1+D^{\varphi_{2} k_{2}}\right) D^{\zeta_{2}}
$$

where $\varphi_{2}$ is the minimum spacing between pair of " 1 "s of permuted weight- 2 information sequence $\mathbf{u}_{2}^{2}, k_{2}=1,2,3,4, \ldots$ and $\zeta_{2}=1,2,3,4, \ldots$ is time delay.

Let the lowest weight of the $i$-th parity sequence is denoted by $z_{\text {min }}^{i}$, which is generated by its corresponding weight-2 information sequence. For any weight-2 information sequence the weight of the $i$-th parity sequence is given as

$$
k_{j}\left(z_{\min }^{i}-2\right)+2
$$

where $k_{j}=1,2,3,4, \ldots, j=1,2$ and $i=1,2$. The weight of an ATC code-word generated by weight- 2 information sequence is given as follows

$$
\begin{aligned}
d & =2+k_{1}\left(z_{\min }^{1}-2\right)+2+k_{2}\left(z_{\min }^{2}-2\right)+2 \\
& =6+k_{1}\left(z_{\min }^{1}-2\right)+k_{2}\left(z_{\min }^{2}-2\right) .
\end{aligned}
$$

Assume that the positions of "1"s are denoted by $f_{1}, f_{2}$ for weight- 2 information sequence $\mathbf{u}_{1}^{2}$. In interleaved information sequence $\mathbf{u}_{2}^{2}$ positions of pair of "1"s are assumed to be $\pi\left(f_{1}\right), \pi\left(f_{2}\right)$. Let us consider a worst scenario for weight-2 information sequence such as

$$
\left|f_{1}-f_{2}\right| \bmod \varphi_{1}=0 \text { and }\left|\pi\left(f_{1}\right)-\pi\left(f_{2}\right)\right| \bmod \varphi_{2}=0 .
$$

In such a situation the separation between the positions of " 1 "s in the information sequence after interleaver mapping is still a multiple of $\varphi_{2}$ as shown in Fig. 4. So for such cases finite number of "1"s are generated by RSC-2 encoder for parity sequence. It results in low weight ATC code-word and degrades the performance for ATC code.

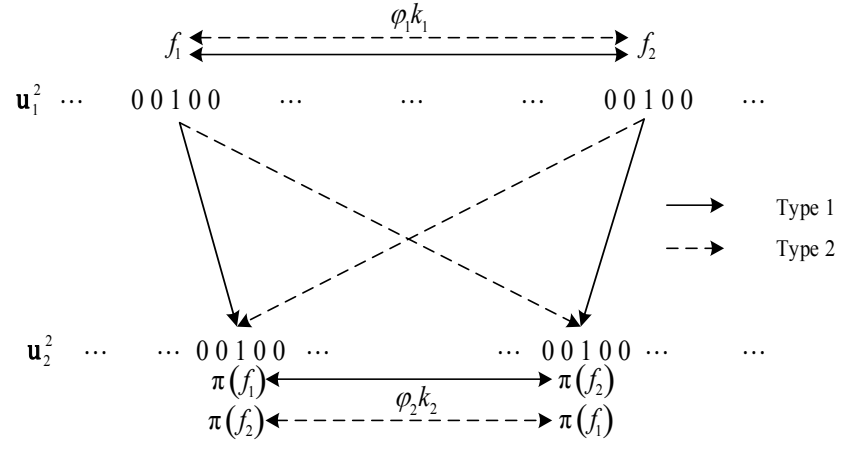

Fig. 4. Weight-2 information sequence is mapped to weight-2 sequence of similar pattern.

To prevent such outcomes, the interleaver must fulfil the mapping conditions given as

$$
\text { If }\left|f_{1}-f_{2}\right| \bmod \varphi_{1}=0 \text { then }\left|\pi\left(f_{1}\right)-\pi\left(f_{2}\right)\right| \bmod \varphi_{2} \neq 0 .
$$

However, not every weight-2 sequence is bad weight sequence. In order to remove only bad weight- 2 sequences usually a parameter $d_{\max }^{\alpha}$ is specified which acts as a threshold, i.e. if the weight of code-word falls below this threshold than that weight- 2 information sequence is removed by interleaving. The relative contribution integral that is used to find a suitable value of $d_{\max }^{\alpha}$ for a particular turbo code is given in [23]. Applying the parameter $d_{\max }^{\alpha=2}$ to (8) results in the following inequality

$$
6+k_{1}\left(z_{\min }^{1}-2\right)+k_{2}\left(z_{\min }^{2}-2\right) \leq d_{\max }^{\alpha=2},
$$

which can be written as

$$
k_{1}\left(z_{\min }^{1}-2\right)+k_{2}\left(z_{\min }^{2}-2\right) \leq d_{\max }^{\alpha=2}-6 .
$$

If $z_{\text {min }}^{1}=z_{\text {min }}^{2}=z_{\min }$ then (12) becomes

$$
\left(k_{1}+k_{2}\right) \leq \frac{d_{\max }^{\alpha=2}-6}{\left(z_{\min }-2\right)} .
$$

If (9) and (12) are satisfied then the weight-2 information sequence $\mathbf{u}_{1}^{2}$ will always produce bad weight ATC codeword. The weight- 3 information sequences which results in low weight ATC code-word can be removed by using $S$ random constraint. The design details of $S$-random interleaver are well documented in [21].

Now we consider weight-4 information sequence which is composed of compound error events, i.e. concatenation of two weight-2 information sequences as depicted in Fig. 5.

The polynomial representation of weight-4 information sequence $\mathbf{u}_{1}{ }_{1}^{4}$ is given as

$$
\mathbf{u}_{1}^{4}(D)=\left(1+D^{\varphi_{1} k_{1}^{\prime}}\right) D^{\zeta_{1}}+\left(1+D^{\varphi_{1} k_{2}^{\prime}}\right) D^{\zeta_{2}}
$$

where $\zeta_{2}>\zeta_{1}+\varphi_{1} k_{1}^{\prime}, k_{1}^{\prime}=1,2,3, \ldots$ and $k_{2}^{\prime}=1,2,3, \ldots$ The polynomial representation of interleaved weight-4 information sequence $\mathbf{u}_{1}{ }^{4}$ is given as

$$
\mathbf{u}_{2}^{4}(D)=\left(1+D^{\varphi_{2} k_{3}^{\prime}}\right) D^{\zeta_{3}}+\left(1+D^{\varphi_{2} k_{4}^{\prime}}\right) D^{\zeta_{4}}
$$




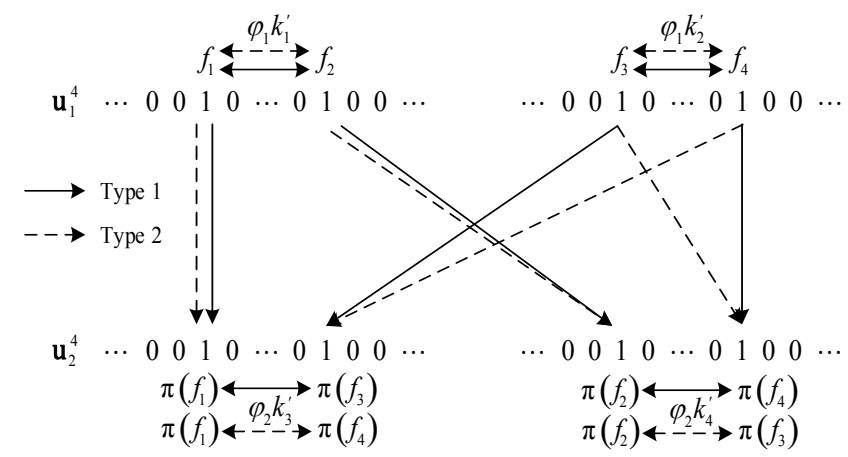

Fig. 5. Weight-4 information sequence is mapped to another weight-4 information sequence after passing through interleaver.

where $\zeta_{4}>\zeta_{3}+\varphi_{2} k_{3}^{\prime}, k_{3}^{\prime}=1,2,3, \ldots$ and $k_{4}^{\prime}=1,2,3, \ldots$ The weight of an ATC code-word generated by weight-4 information sequence is given as follows

$$
\begin{gathered}
d=4+\alpha\left(\mathbf{v}_{1}\right)+\alpha\left(\mathbf{v}_{2}\right) \\
d=12+\left(k_{1}^{\prime}+k_{2}^{\prime}\right)\left(z_{\min }^{1}-2\right)+\left(k_{3}^{\prime}+k_{4}^{\prime}\right)\left(z_{\text {min }}^{2}-2\right) .
\end{gathered}
$$

Assume that the positions of " 1 "s are denoted by $f_{1}, f_{2}$, $f_{3}$ and $f_{4}$ for weight- 4 information sequence $\mathbf{u}_{1}^{4}$, where $f_{1}<f_{2}<f_{3}<f_{4}$. In interleaved weight- 4 information sequence $\mathbf{u}_{2}^{4}$ positions of " 1 "s are assumed to be $\pi\left(f_{1}\right), \pi\left(f_{2}\right)$, $\pi\left(f_{3}\right)$, and $\pi\left(f_{4}\right)$. The detrimental scenario for weight-4 information sequence is given as

$$
\left\{\begin{array}{l}
\text { If }\left|f_{1}-f_{2}\right| \bmod \varphi_{1}=0 \text { and }\left|f_{3}-f_{4}\right| \bmod \varphi_{1}=0 \\
\text { then }\left|\pi\left(f_{1}\right)-\pi\left(f_{3}\right)\right| \bmod \varphi_{2}=0 \\
\text { and }\left|\pi\left(f_{2}\right)-\pi\left(f_{4}\right)\right| \bmod \varphi_{2}=0
\end{array}\right.
$$

or

$$
\left\{\begin{array}{l}
\text { If }\left|f_{1}-f_{2}\right| \bmod \varphi_{1}=0 \text { and }\left|f_{3}-f_{4}\right| \bmod \varphi_{1}=0 \\
\text { then }\left|\pi\left(f_{1}\right)-\pi\left(f_{4}\right)\right| \bmod \varphi_{2}=0 \\
\text { and }\left|\pi\left(f_{2}\right)-\pi\left(f_{3}\right)\right| \bmod \varphi_{2}=0 .
\end{array}\right.
$$

The URI again maps the weight-4 information sequence as a concatenation of two weight- 2 error events as shown in Fig. 5, which results in bad weight parity sequence and generates low weight ATC code-word. Such concatenated events can be avoided if the interleaver satisfies the following mapping conditions

$$
\left\{\begin{array}{l}
\text { If }\left|f_{1}-f_{2}\right| \bmod \varphi_{1}=0 \text { and }\left|f_{3}-f_{4}\right| \bmod \varphi_{1}=0 \\
\text { then }\left|\pi\left(f_{1}\right)-\pi\left(f_{3}\right)\right| \bmod \varphi_{2} \neq 0 \\
\text { and }\left|\pi\left(f_{2}\right)-\pi\left(f_{4}\right)\right| \bmod \varphi_{2} \neq 0
\end{array}\right.
$$

$$
\left\{\begin{array}{l}
\text { If }\left|f_{1}-f_{2}\right| \bmod \varphi_{1}=0 \text { and }\left|f_{3}-f_{4}\right| \bmod \varphi_{1}=0 \\
\text { then }\left|\pi\left(f_{1}\right)-\pi\left(f_{4}\right)\right| \bmod \varphi_{2} \neq 0 \\
\text { and }\left|\pi\left(f_{2}\right)-\pi\left(f_{3}\right)\right| \bmod \varphi_{2} \neq 0 .
\end{array}\right.
$$

All weight- 2 information sequences that produce finite weight are not bad sequences in general. Similarly, all weight-4 information sequences are not bad sequences either. In order to remove only bad weight- 4 sequences a parameter $d_{\max }^{\alpha=4}$ is specified. This parameter acts like a threshold and its functionality is similar to $d_{\max }^{\alpha=2}$ parameter. Applying this parameter $d_{\max }^{\alpha=4}$ to weight-4 sequences will result in the following inequality

$$
\left(12+\left(k_{1}^{\prime}+k_{2}^{\prime}\right)\left(z_{\min }^{1}-2\right)+\left(k_{3}^{\prime}+k_{4}^{\prime}\right)\left(z_{\min }^{2}-2\right)\right) \leq d_{\max }^{\alpha=4}
$$

which can be written as

$$
\left(\left(k_{1}^{\prime}+k_{2}^{\prime}\right)\left(z_{\min }^{1}-2\right)+\left(k_{3}^{\prime}+k_{4}^{\prime}\right)\left(z_{\min }^{2}-2\right)\right) \leq d_{\max }^{\alpha=4}-12 .
$$

If $z_{\text {min }}^{1}=z_{\text {min }}^{2}=z_{\min }$ then (21) becomes

$$
\left(k_{1}^{\prime}+k_{2}^{\prime}+k_{3}^{\prime}+k_{4}^{\prime}\right) \leq \frac{d_{\max }^{\alpha=4}-12}{\left(z_{\min }-2\right)} \text {. }
$$

To check whether the weight-4 information sequence $\mathbf{u}_{1}{ }_{1}^{4}$ is producing bad weight ATC code-word, the conditions in (18) and (21) should be satisfied.

The $S$-random constraint is sufficient for breaking the information sequences having $\alpha>4$ [31]. Therefore, it is not required to consider each of them separately in the design of interleaver. Thus deploying CMI is considered as an optimum choice of interleaver as the mapping conditions for weight-2, 3 and 4 are satisfied in its design.

\section{Spatial NLMS Adaptive Filter}

The multiple receive antennas are usually deployed at the destination node that not only mitigates the harsh effects of fading channel but also achieves receive diversity. The source and relay nodes can be thought of mobile terminals while the destination node behaves like a base terminal. As the base terminals usually do not have power constraint therefore multiple receive antennas at the base terminal seems to be a quite reasonable assumption in our system model.

We have assumed an ideal coded-cooperation scenario, i.e. $S-R_{\omega}$ channel is ideal. Furthermore, the destination node does not have any prior knowledge of source to destination $(S-D)$ and relays to destination $(R-D)$ channels. Therefore, in order to estimate $S-D$ and $R-D$ channels, adaptive spatial NLMS [24] filters must be employed at the destination node. In this paper, two spatial NLMS filters are deployed to estimate the unknown aforementioned channels. As in the second time slot, all relay nodes transmit their signals to their corresponding receive antennas at the destination node. Since no co-channel interference is considered at the destination node during the second time slot, therefore employing a spatial NLMS filters is a reasonable option to estimate the aforementioned channels.

The $k$-th received sequence $\boldsymbol{\delta}_{k}^{l}$ during the $l$-th time slot for $L$ receiving antennas are given by 


$$
\boldsymbol{\delta}_{k}^{l}=\mathbf{H}^{l} a_{k}^{l}+\mathbf{n}_{k}^{l}
$$

where

$$
\begin{gathered}
\boldsymbol{\delta}_{k}^{l}=\left[\begin{array}{llll}
\delta_{k, 1}^{l} \delta_{k, 2}^{l} & \ldots & \delta_{k, L}^{l}
\end{array}\right]^{\mathrm{T}}, \\
\mathbf{n}_{k}^{l}=\left[\begin{array}{llll}
n_{k, 1}^{l} n_{k, 2}^{l} & \ldots & n_{k, L}^{l}
\end{array}\right]^{\mathrm{T}}, \\
\mathbf{H}^{l}=\left[\begin{array}{lll}
h_{1}^{l} h_{2}^{l} & \ldots . & h_{L}^{l}
\end{array}\right]^{\mathrm{T}}
\end{gathered}
$$

where $\mathbf{H}^{l}$ is the memory-less Rayleigh block fading channel vector with zero mean and unit variance, $a_{k}^{l}$ is the QPSK modulated symbol, $\mathbf{n}_{k}^{l}$ is the independent AWGN with zero mean and $N_{0}$ variance for the $l$-th time slot, $l=1,2$ for source and relay nodes, respectively.

The spatial NLMS transversal filter for the $l$-th time slot is shown in Fig. 6. The difference between the transmitted bit $s_{k}^{l}$ of the training sequence and the filtered output $y_{k}^{l}$ is termed as an error signal and is given as follows

$$
\xi_{k}^{l}=s_{k}^{l}-\left(\mathbf{b}^{l}\right)^{\mathrm{T}} \boldsymbol{\delta}_{k}^{l}
$$

where $\mathbf{b}^{l}=\left[b_{1}^{l} b_{2}^{l} \ldots b_{L}^{l}\right]^{\mathrm{T}}$ is the transversal filter vector.

The absolute square of error signal is given as follows

$$
\left|\xi_{k}^{l}\right|^{2}=\left|s_{k}^{l}\right|^{2}-2 \operatorname{Re}\left[s_{k}^{l}\left\{\left(\mathbf{b}^{l}\right)^{*} \overline{\boldsymbol{\delta}}_{k}^{l}\right\}\right]+\left(\mathbf{b}^{l}\right)^{*}\left[\overline{\boldsymbol{\delta}}_{k}^{l}\left(\boldsymbol{\delta}_{k}^{l}\right)^{\mathrm{T}}\right] \mathbf{b}^{l} .
$$

The gradient of $\left|\xi_{k}^{l}\right|^{2}$ w.r.t $\mathbf{b}$ is given by

$$
\Delta_{b}\left(\left|\xi_{k}^{l}\right|^{2}\right)=2\left[\overline{\boldsymbol{\delta}}_{k}^{l}\left(\boldsymbol{\delta}_{k}^{l}\right)^{\mathrm{T}}\right] \mathbf{b}^{l}-2 s_{k}^{l} \overline{\boldsymbol{\delta}}_{k}^{l}
$$

The adaptive weights of filter are

$$
\mathbf{b}_{k+1}^{l}=\mathbf{b}_{k}^{l}-\left.\frac{\mu_{k}^{l}}{2} \Delta_{b}\left(\left|\xi_{k}^{l}\right|^{2}\right)\right|_{\mathbf{b}^{\prime}=\mathbf{b}_{k}^{l}} .
$$

To compute the updated weight vector of the filter, the step-size becomes

$$
\mu_{k}^{l}=\frac{\rho}{c+\left\|\boldsymbol{\delta}_{k}^{l}\right\|^{2}}
$$

where $\mu_{k}^{l}$ is the step-size parameter at the $k$-th sample, $\rho$ is the normalized step-size $(0<\rho<2)$ and $c$ defines the safety factor.

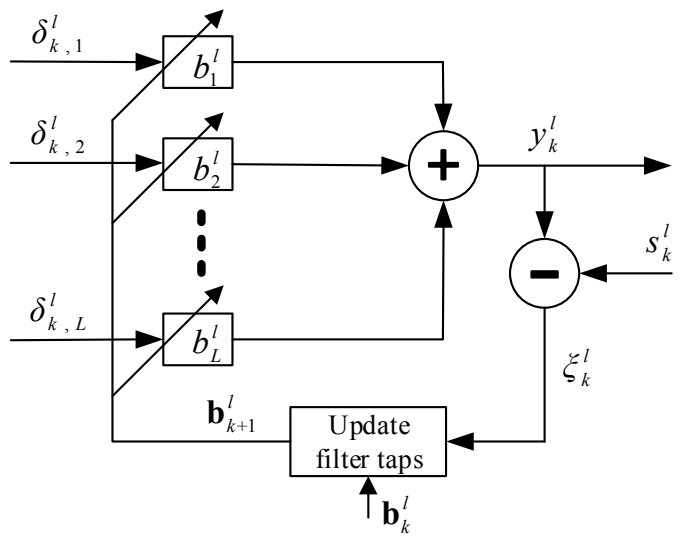

Fig. 6. Spatial NLMS adaptive filter for the $l$-th time slot.
The step-size is an important parameter of NLMS filter as it affects the convergence rate and the learning curve of a filter. The power of input signal changes with time, which causes the step-size to fluctuate. The small power signals will make the convergence rate slow, however, high power signals will cause convergence rate to speed up and eventually result in more error. Therefore, in order to cope in such a situation, the step-size parameter is adjusted according to the power of input signal and is usually taken as a normalized value. Sometimes $\boldsymbol{\delta}_{k}^{l}$ is very small and updating weight vector becomes difficult, so a small positive constant $c$ known as a safety factor $(0<c<1)$ is applied to overcome this uncertainty.

The number of iterations for estimating the channel depends on the length of the training sequence. Let $K_{\mathrm{T}}$ be the length of training sequence and the channel will be estimated after $K_{\mathrm{T}}$ iterations. The estimated channel is given by

$$
\hat{\mathbf{H}}^{l}=\mathbf{b}_{K_{\mathrm{T}}}^{l}=\left[\begin{array}{lll}
b_{1}^{l} & b_{2}^{l} \ldots b_{L}^{l}
\end{array}\right]^{\mathrm{T}}
$$

where $l=1,2$ for $S-D$ and $R-D$ channels, respectively.

\section{Soft Demodulation}

Soft-demodulation for MIMO is proposed in [32]. The soft-demodulator with multi-receive antennas and joint iterative SISO decoder are connected in serial concatenation. The soft-demodulator takes a-priori LLRs of coded bits and channel observations $\boldsymbol{\delta}_{k}^{l}$ Then it generates aposteriori LLRs $\lambda_{D, i}^{l}$ for each coded bit under the assumption that the channel observations are independent of each other. For the $i$-th element of the $k$-th transmitted bit-vector $q_{k, i}$, the soft-demodulator calculates

$$
\lambda_{D, k, i}^{l}=\ln \frac{P\left\{q_{k, i}=+1 \mid \boldsymbol{\delta}_{k}^{l}\right\}}{P\left\{q_{k, i}=-1 \mid \boldsymbol{\delta}_{k}^{l}\right\}} .
$$

For simplicity, we remove $k$ from the subscript and $l$ from the superscript. Using Bayes' rule, (33) can be written as

$$
\lambda_{D, i}=\ln \frac{\sum_{\Psi_{i,+1}} p(\boldsymbol{\delta} \mid \mathbf{q}) p(\mathbf{q})}{\sum_{\Psi_{i,-1}} p(\boldsymbol{\delta} \mid \mathbf{q}) p(\mathbf{q})}
$$

where $\boldsymbol{\Psi}=\left\{\mathbf{q} \in\{-1,+1\}^{2}\right\}$ represents a set of all possible combinations of transmitted bit-vectors and $\Psi_{i, \mp 1}=\left\{\mathbf{q} \in \Psi \mid q_{i}= \pm 1\right\}$ denotes the set of bit vectors $\mathbf{q}$ having $q_{i}= \pm 1$. The likelihood function $p(\boldsymbol{\delta} \mid \mathbf{q})$ in (34) can be expressed as

$$
p(\boldsymbol{\delta} \mid \mathbf{q}) \propto \exp \left(-\|\boldsymbol{\delta}-\mathbf{H} \mathcal{M}(\mathbf{q})\|^{2} / 2 \sigma^{2}\right) .
$$

Since the elements of $\mathbf{q}$ are considered as independent, the a-priori probability $p(\mathbf{q})$ can be calculated as follows

$$
p(\mathbf{q}) \approx \prod_{i} p\left(q_{i}\right)
$$




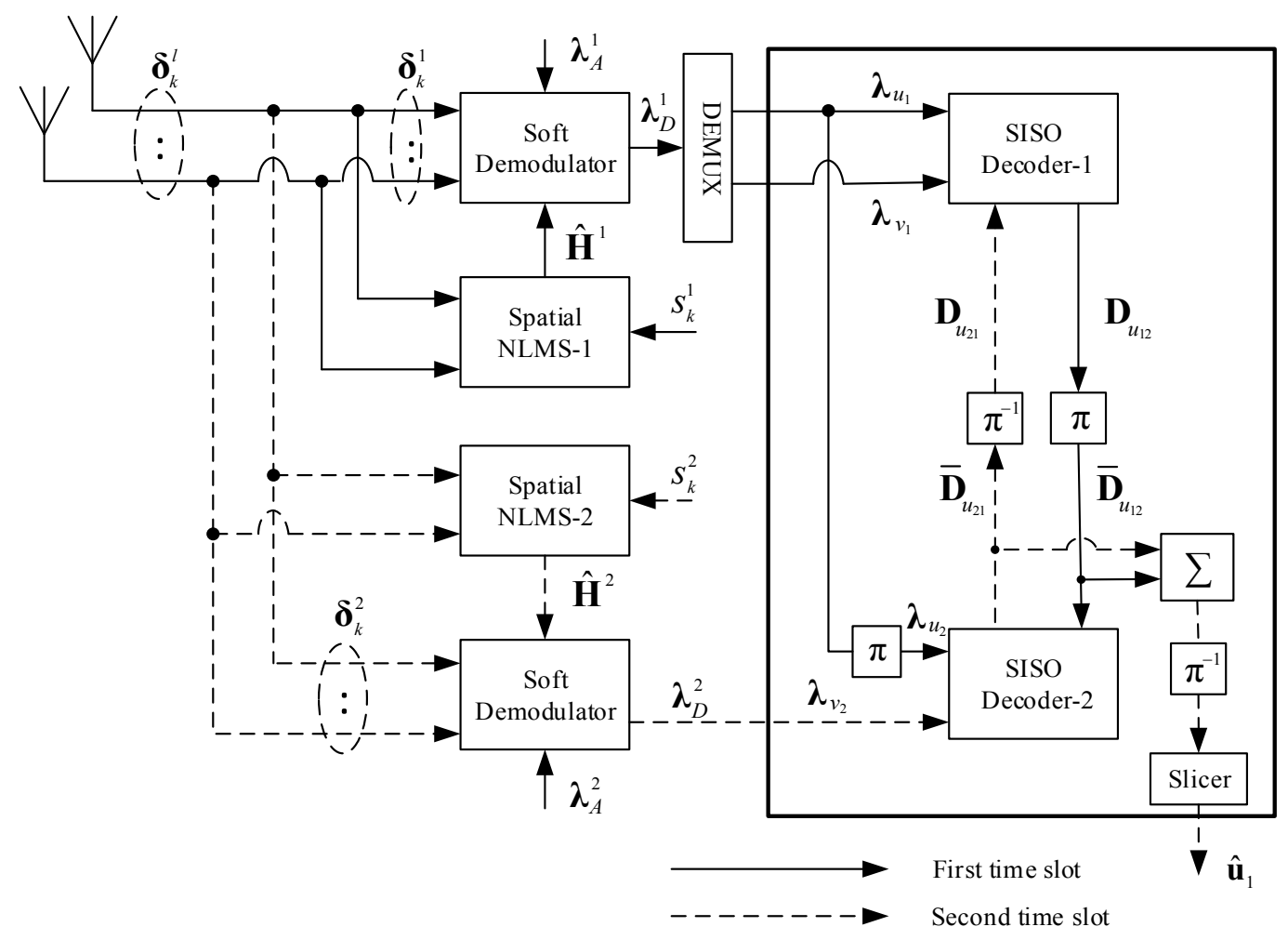

Fig. 7. Joint iterative SISO decoding for DATC scheme.

The a-priori LLR is defined as

$$
\lambda_{A, i}=\ln \left(\frac{p\left(q_{i}=+1\right)}{p\left(q_{i}=-1\right)}\right) .
$$

If $\lambda_{\mathrm{A}}$ represents the vector of a-priori LLR, then $p(\mathbf{q})$ can be represented as

$$
\prod_{i} p\left(q_{i}\right) \propto \exp \left(\lambda_{\mathrm{A}}^{\mathrm{T}} \mathbf{q} / 2\right)
$$

Using (35) and (38), the expression in (34) can be written as

$$
\lambda_{D, i} \cong \ln \frac{\sum_{\Psi_{i,+1}} \exp \left(-W(\mathbf{q}) /\left(2 \sigma^{2}\right)\right)}{\sum_{\Psi_{i,-1}} \exp \left(-W(\mathbf{q}) /\left(2 \sigma^{2}\right)\right)}
$$

where

$$
W(\mathbf{q}) \triangleq\|\boldsymbol{\delta}-\mathbf{H} \mathcal{M}(\mathbf{q})\|_{2}^{2}-\sigma^{2} \lambda_{\mathrm{A}}^{\mathrm{T}} \mathbf{q}
$$

The demodulator uses (39) to generate LLR for coded bits. The complexity of the demodulator increases with increase in number of elements in the set $\Psi_{i, \mp 1}$.

\section{Joint Iterative SISO Decoding for DATC Scheme}

The joint iterative SISO decoding for DATC scheme is shown in Fig. 7. The SISO module for iterative decoding of serial and parallel concatenated turbo codes has been discussed in literature [33]. The joint decoder consists of two different SISO decoders. The SISO decoder consists of three inputs and one output. A-priori probabilities of systematic bits and parity bits are given as input to SISO decoder which results in extrinsic information as an output.

During the first time slot, the soft demodulator generates LLRs $\lambda_{D}^{1}$, these LLRs are soft-bits corresponding to the systematic bits and parity bits which were transmitted from the source node. The de-multiplexer breaks the LLRs $\lambda_{D}^{1}$ into two separate streams of systematic LLRs $\lambda_{u_{1}}$ and parity LLRs $\lambda_{\nu_{1}}$. These LLRs are received by SISOdecoder-1 which outputs the extrinsic information $\mathbf{D}_{u_{12}}$. The extrinsic information of systematic bits $\mathbf{D}_{u_{12}}$ is interleaved and given as a-priori input to SISO-decoder-2.

In the second time slot, the LLRs $\lambda_{D}^{2}$ are generated by the soft demodulator. These LLRs are soft-bits corresponding to the parity bits of RSC-2 encoder which were transmitted from relay node. The extrinsic information $\overline{\mathbf{D}}_{u_{21}}$ is deinterleaved to form extrinsic information $\mathbf{D}_{u_{21}}$. This extrinsic information $\mathbf{D}_{u_{21}}$ is given back to SISO-decoder-1. The extrinsic information is interchanged between SISOdecoders for a specific number of iterations. In order to get the estimated information sequence $\hat{\mathbf{u}}_{1}$, the extrinsic information $\overline{\mathbf{D}}_{u_{21}}$ and $\overline{\mathbf{D}}_{u_{12}}$ are summed up and passed through a slicer after de-interleaving.

\section{Simulated Results and Discussion}

This section discusses the simulation results of ATC and the proposed DATC coded-cooperative scheme with 
CMI and URI. The ATC scheme is simulated over AWGN and Rayleigh fading channels while the DATC scheme is simulated over Rayleigh fading channels with multi-receive antenna and unknown CSI at the destination node. In order to have fair comparison between the coded-cooperative scheme and non-cooperative scheme with CMI, overall code rate at the destination node is kept the same, i.e. $R_{\mathrm{c}}=1 / 3$. The generator matrix for ATC and DATC is $\mathbf{G}(13 / 15,11 / 15)_{8}$. The length of CMI and URI is $K=1024$. The channels are assumed to be slow Rayleigh fading channels. For all coded-cooperative simulations, the $S-R_{\omega}$ channel is assumed to be ideal $\left(\mathrm{SNR}_{S R_{\omega}}=\infty\right)$. The relay nodes are placed near to the destination node in order to get the advantage of SNR over the source node. In case of two relay nodes, one relay node has given $1 \mathrm{~dB}$ gain in SNR while the other relay node has given $2 \mathrm{~dB}$ gain in SNR over the source node. Furthermore, in the case of three relay nodes, two relay nodes have given $2 \mathrm{~dB}$ gain in SNR while one relay node has given $1 \mathrm{~dB}$ gain in SNR over the source node. Moreover, spatial NLMS adaptive filters, soft demodulators and joint SISO decoder are employed at the destination node. The SNR per bit is used for all simulations.

\subsection{Performance Comparison between Conventional STC and ATC over AWGN Channel}

The BER performance comparison between conventional STC and proposed ATC having generator matrices $\mathbf{G}(11 / 15,11 / 15)_{8}$ and $\mathbf{G}(13 / 15,11 / 15)_{8}$, respectively over AWGN channel with QPSK modulation is shown in Fig. 8. The information block length is considered to be $K=400$ bits. The BER curve of ATC with URI clearly shows that it outperforms conventional STC with URI over the whole SNR range. The performance gain of ATC with URI at $\mathrm{BER} \approx 3 \times 10^{-6}$ over conventional STC with URI is about $0.5 \mathrm{~dB}$.

It can be seen that by eliminating low weight codewords from ATC using CMI, the performance in terms of BER improves significantly. ATC with CMI achieves approx. $0.4 \mathrm{~dB}$ gain as compared to ATC with URI at $\mathrm{BER} \approx 3 \times 10^{-6}$. The performance gain of ATC is also improved by using CMI in comparison to conventional STC with URI. At BER $\approx 3 \times 10^{-6}$, ATC with CMI has the performance gain of about $0.9 \mathrm{~dB}$ over conventional STC with URI.

\subsection{Performance Comparison between CMI and URI for ATC Non-Cooperation with Multi-Receive Antennas}

In this subsection, we consider the ATC non-cooperation with CMI and URI under perfect and imperfect CSIs and different number of receive antennas. The spatial NLMS adaptive filter is adopted for channel estimation at the destination node. The length of training sequence is 40

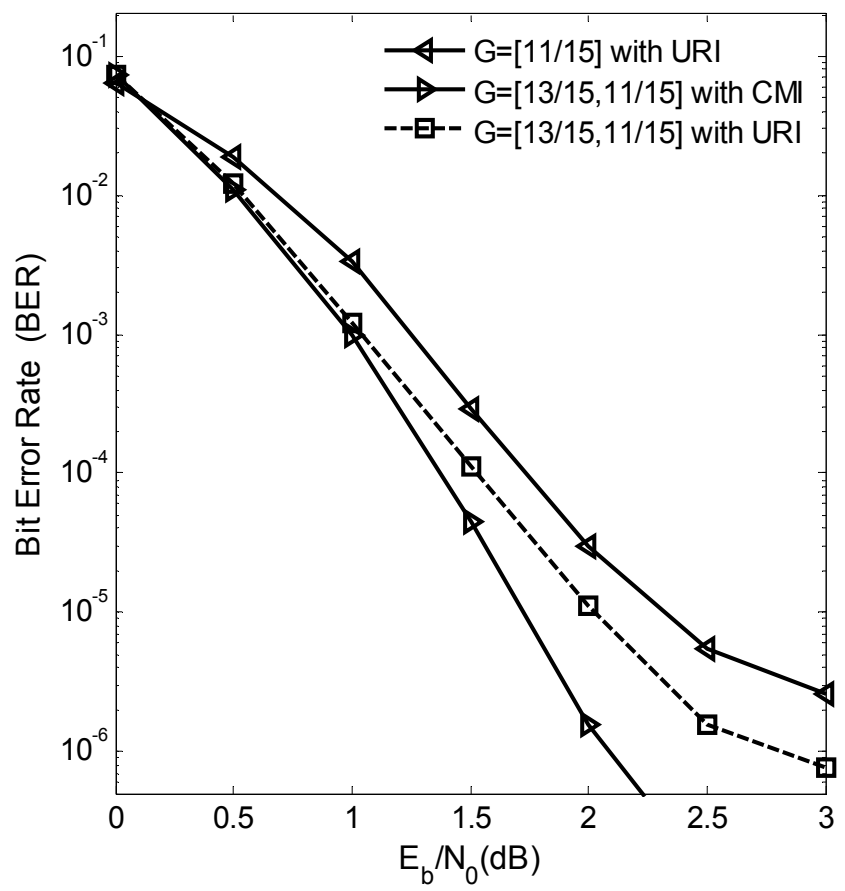

Fig. 8. BER performance of conventional STC and ATC over AWGN channel, $K=400$ and 8 decoding iterations.

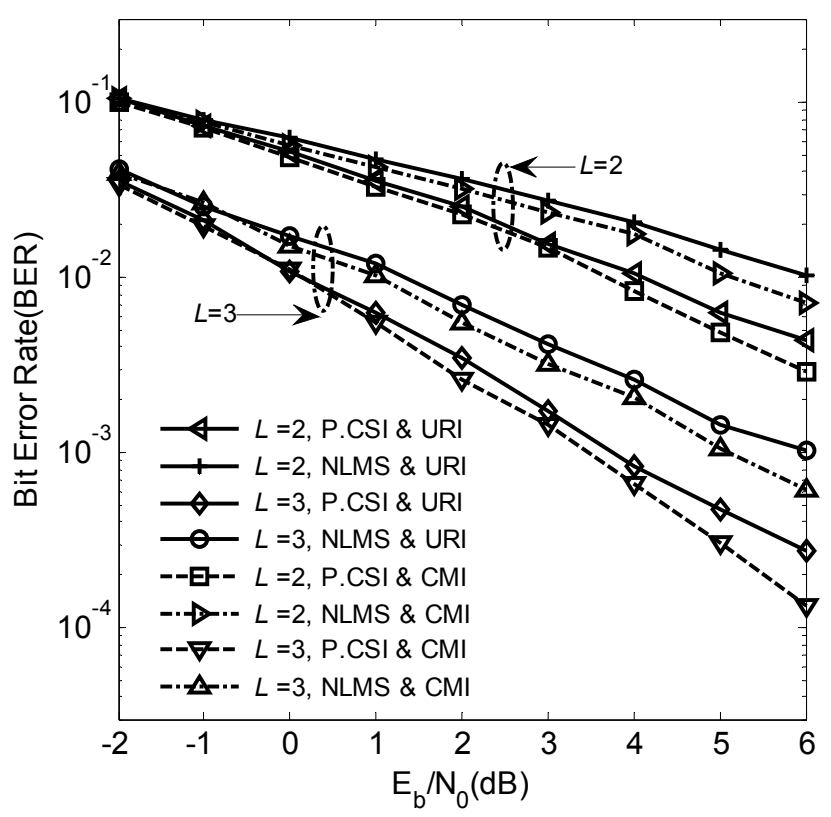

Fig. 9. BER performance of ATC non-cooperation with CMI and URI over perfect / imperfect CSI (Rayleigh fading), 8 decoding iterations, $K=1024$ and different number of receive antennas at the destination.

for spatial NLMS filter. Safety parameter has constant value 0.05 and step-size parameter $\mu_{k}$ at the $k$-th sample is $0.1 /\left(0.05+\left\|\boldsymbol{\delta}_{k}\right\|^{2}\right)$.

The BER performance comparison between CMI and URI for ATC non-cooperation with multiple receive antennas is shown in Fig. 9. It can be observed that there is a narrow gap between BER performances of CMI and URI for ATC non-cooperation under perfect CSI. At SNR of $5 \mathrm{~dB}$, the BER performance under $\mathrm{CMI}$ is $5 \times 10^{-3}$ and under 
URI is $6 \times 10^{-3}$ for two receive antennas. Similarly at SNR of $5 \mathrm{~dB}$, the BER performances are $3 \times 10^{-4}$ and $4.5 \times 10^{-4}$ for CMI and URI, respectively in case of three receive antennas.

It can be seen that there is also a small gap between BER performances of CMI and URI for ATC non-cooperation under estimated CSI by spatial NLMS filter. For instance, the performance gain of $0.4 \mathrm{~dB}$ is achieved at $\mathrm{BER} \approx 1.5 \times 10^{-2}$ for two receive antennas. Similarly at $\mathrm{BER} \approx 1.5 \times 10^{-3}$, the performance gain of $0.5 \mathrm{~dB}$ is observed for the case of three receive antennas. The BER performance gap between perfect CSI and estimated CSI for various receive antennas are under acceptable range. This gap can further be minimized by increasing the length of training sequence but it adds some latency to the system.

The receive diversity is increased by increasing the receive antennas at the destination node. The gain ranges from 4 to $4.5 \mathrm{~dB}$ between two and three receive antennas over perfect $\mathrm{CSI}$ at $\mathrm{BER} \approx 4 \times 10^{-3}$. Similarly the gain ranges from 4 to $5 \mathrm{~dB}$ for estimated CSI between two and three receive antennas at $\mathrm{BER} \approx 10^{-2}$.

\subsection{Performance Comparison of DATC Coded-Cooperation $\left(\mathrm{SNR}_{S R_{\omega}}=\infty\right)$ and ATC Non-Cooperation with CMI and Multi-Receive Antennas over Perfect and Imperfect CSI}

In this subsection, the simulated results of DATC in coded-cooperation scenario incorporating CMI are presented. As evident from the Monte Carlo simulation results shown in Fig. 10, the proposed scheme outperforms its corresponding non-cooperation scheme under identical conditions.

For the case of two relay nodes, DATC coded-cooperation scheme gives $2.7 \mathrm{~dB}$ performance gain over ATC non-cooperation scheme with two receive antennas at $\mathrm{BER} \approx 3 \times 10^{-3}$ over perfect CSI. Similarly the BER performance gain of $3.5 \mathrm{~dB}$ is observed for three relay nodes at $\mathrm{BER} \approx 0.5 \times 10^{-4}$ over perfect CSI.

In case of estimated CSI for multi-relay DATC coded cooperation scheme, two spatial NLMS adaptive filters are adopted for channel estimation at the destination node. The length of training sequence, safety and step-size parameters are all kept the same as for coded non-cooperation scheme. At $\mathrm{BER} \approx 7 \times 10^{-3}$, under imperfect CSIs and two receive antennas, the two relays DATC coded cooperation scheme achieves the performance gain of $3 \mathrm{~dB}$ over ATC non-cooperation scheme. Moreover, the gain of $3.3 \mathrm{~dB}$ is observed for three relay nodes at $\mathrm{BER} \approx 6 \times 10^{-4}$.

The BER performance of three relays DATC scheme is better than two relays DATC scheme under identical conditions. Increasing the number of relay nodes improves the information reliability by providing diversity and hence the BER performance is improved. At SNR of $6 \mathrm{~dB}$, the

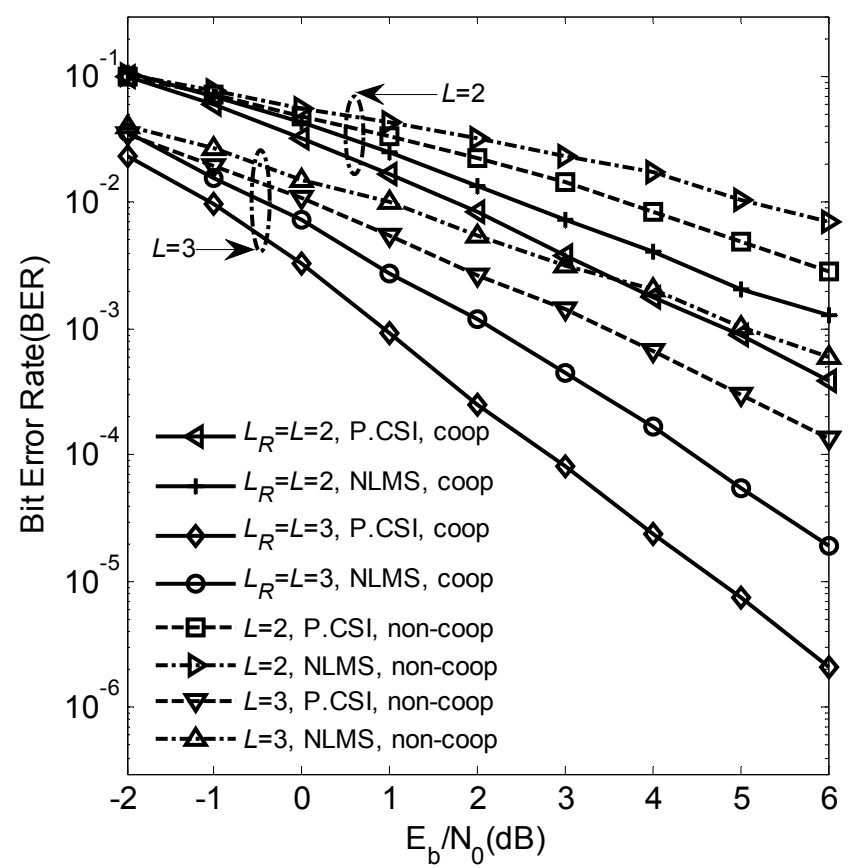

Fig. 10. BER performance of multi-relay DATC ideal codedcooperation and non-cooperation with CMI over perfect / imperfect CSI (Rayleigh fading), eight decoding iterations, $K=1024$ and different number of receive antennas $L$ at the destination.

BER performance of multi-relay DATC scheme with two and three relay nodes are $4 \times 10^{-4}$ and $2 \times 10^{-6}$, respectively over perfect CSI. Similarly in case of imperfect CSI, the BER performance $0.5 \times 10^{-3}$ and $2 \times 10^{-5}$ for two and three relay nodes, respectively is observed at $6 \mathrm{~dB}$.

The better BER performance in the case of multi-relays is based on the facts that there is an increase in SNR due to the placement of additional relays near to destination. Moreover, deploying multi-relays causes additional spatial diversity gain. However, it is worth mentioning that the proposed coded-cooperation scheme introduces extra latency in the whole communication system as compared to non-cooperation scheme. In addition it also requires extra spatial NLMS filter which results in increased hardware complexity at the destination.

\section{Conclusion}

In this paper, we have analyzed the BER performance of ATC $\mathbf{G}(13 / 15,11 / 15)_{8}$ on the basis of CMI and URI over AWGN channel. It is concluded that the performance of ATC with CMI outperforms ATC with URI under identical conditions. The proposed ATC with CMI is also compared with its counterpart STC G(11/15, 11/15) 8 . The simulated results have shown that the performance of ATC is quite superior over conventional turbo code. Moreover, the proposed ATC is also examined under perfect and imperfect CSI with multi-receive antennas over the Rayleigh block fading channel on the basis of CMI and URI. Our proposed schemes incorporate both coding and diversity gains. In addition, it is also reinforced by Monte Carlo 
simulations that CMI is always an optimum choice of interleaver in the design of turbo code. The CMI is further deployed in coded-cooperation scenario with multiple relays under the proposed encoding schemes. Finally, the BER performance of the coded-cooperation under the proposed encoding scheme is analyzed, and compared with its corresponding non-cooperation counterpart scheme. The coded-cooperation scheme outperforms the non-cooperation scheme under identical conditions by a significant gain of 2.7 to $3.5 \mathrm{~dB}$.

\section{Acknowledgments}

The authors would like to thank the Editor and anonymous reviewers for their precious suggestions, comments and reviews that help in improving the quality of this paper.

\section{References}

[1] BARRY, J. R., LEE, E. A., MESSERSCHMITT, D. G. Digital Communication. $3^{\text {rd }}$ ed. Springer US, 2004. ISBN: 978-1-4615$0227-2$

[2] FOSCHINI, G. J., GANS, M. J. On limits of wireless communications in a fading environment when using multiple antennas. Wireless Personal Communications, 1998, vol. 6, no. 3, p. 311-335. DOI: $10.1023 / \mathrm{A}: 1008889222784$

[3] NOSRATINIA, A., HUNTER, T. E., HEDAYAT, A. Cooperative communication in wireless networks. IEEE Communications Magazine, 2004, vol. 42, no. 10, p. 74-80. DOI: $10.1109 /$ mcom.2004.1341264

[4] VAN DER MEULEN, E. C. Three-terminal communication channels. Advances in Applied Probability, 1971, vol. 3, no. 1, p. $120-154$. DOI: $10.2307 / 1426331$

[5] LANEMAN, J. N., WORNELl, G. W., TSE, D. N. An efficient protocol for realizing cooperative diversity in wireless networks. In Proceedings of IEEE International Symposium on Information Theory, 2001, p. 294. DOI: 10.1109/ISIT.2001.936157

[6] AVESTIMEHR, A. S., DigGAVI, S. N., DAVID, N. Wireless network information flow: A deterministic approach. IEEE Transactions on Information Theory, 2011, vol. 57, no. 4, p. 1872 to 1905. DOI: 10.1109/TIT.2011. 2110110

[7] COVER, T., GAMAL, A. E. Capacity theorems for the relay channel. IEEE Transactions on Information Theory, 1979, vol. 25, no. 5, p. 572-584. DOI: 10.1109/TIT.1979.1056084

[8] SENDONARIS, A., ERKIP, E., AAZHANG, B. User cooperation diversity. Part I. System description. IEEE Transactions on Communications, 2003, vol. 51, no. 11, p. 1927-1938. DOI: 10.1109/TCOMM.2003.818096

[9] HUNTER, T. E., NOSRATINIA, A. Cooperation diversity through coding. In Proceedings of IEEE International Symposium on Information Theory. Lausanne (Switzerland), 2002, p. 220. DOI: 10.1109/ISIT.2002.1023492

[10] SOLIMAN, T. H., YANG, F., EJAZ, S. Interleaving gains for receive diversity schemes of distributed turbo codes in wireless half-duplex relay channels. Radioengineering, 2015, vol. 24, no. 2, p. 481-488. DOI: 10.13164/re.2015. 0481

[11] HU, J., DUMAN, T. M. Low density parity check codes over wireless relay channels. IEEE Transactions on Wireless
Communications, 2007, vol. 6, no. 9, p. 3384-3394. DOI: 10.1109/TWC.2007.06083

[12] ELFITURI, M., HAMOUDA, W., GHRAYEB, A. A convolutional-based distributed coded cooperation scheme for relay channels. IEEE Transactions on Vehicular Technology, 2009, vol. 58, no. 2, p. 655-669. DOI: 10.1109/TVT.2008.927033

[13] EJAZ, S.,YANG, F.-F. Jointly optimized Reed-Muller cCodes for multi-level multi-relay coded-cooperative VANETS. IEEE Transactions on Vehicular Technology, 2017, vol. 66, no. 5, p. 4017-4028. DOI: 10.1109/TVT.2016.2604320

[14] SOLIMAN, T. H., YANG, F. Cooperative Punctured Polar Coding (CPPC) scheme based on Plotkin's construction. Radioengineering, 2016, vol. 25 , no. 3, p. 482-489. DOI: 10.13164/re.2016.0482

[15] BRAVO-SANTOS, A. Polar codes for the Rayleigh fading channel. IEEE Communications Letters, 2013, vol. 17, no. 12, p. 2352-2355. DOI: 10.1109/LCOMM.2013.111113. 132103

[16] EJAZ, S., YANG, F.-F. Turbo codes with modified code matched interleaver for coded-cooperation in half-duplex wireless relay networks. Frequenz, 2015, vol. 69, p. 171-184. DOI: $10.1515 /$ freq-2014-0072

[17] KIM, H. Coding and modulation techniques for high spectral efficiency transmission in $5 \mathrm{G}$ and satcom. In 23rd European Signal Processing Conference (EUSIPCO), 2015, p. 2746-2750. DOI: 10.1109/EUSIPCO.2015. 7362884

[18] PereZ, L. C., SEghers, J., COSTello, D. J. A distance spectrum interpretation of turbo codes. IEEE Transactions on Information Theory, 1996, vol. 42, no. 6, p. 1698-1709. DOI: $10.1109 / 18.556666$

[19] HALL, E. K., WILSON, S. G. Convolutional interleavers for stream-oriented parallel concatenated convolutional codes. In IEEE International Symposium on Information Theory, 1998, p. 33. DOI: $10.1109 /$ ISIT.1998.708613

[20] RAMSEY, J. Realization of optimum interleavers. IEEE Transactions on Information Theory, 1970, vol. 16, no. 3, p. 338 to 345. DOI: 10.1109/TIT.1970.1054443

[21] DIVSAlaR, D., POLlaRA, F. Turbo codes for PCS applications. In IEEE International Conference on Communications, ICC'95 Seattle, Gateway to Globalization, 1995, vol. 1, p. 54-59. DOI: 10.1109/ICC.1995.525138

[22] FENG, W., YUAN, J., VUCETIC, B. S. A code-matched interleaver design for turbo codes. IEEE Transactions on Communications, 2002, vol. 50, no. 6, p. 926-937. DOI: 10.1109/TCOMM.2002.1010612

[23] RAMASAMY, K., SIDDIQI, M. U. A new class of asymmetric turbo code for $3 \mathrm{G}$ systems. AEU-International Journal of Electronics and Communications, 2006, vol. 60, no. 6, p. 447-458. DOI: 10.1016/j.aeue.2005.09.007

[24] JOY, A., CHAKKA, V. Performance comparison of LMS/NLMS based transceiver filters for MIMO two-way relaying scheme. In International Conference on Communications and Signal Processing (ICCSP). 2011, p. 105-107. DOI: 10.1109/ICCSP.2011.5739411

[25] CATTIVELli, F. S., SAYED, A. H. Analysis of spatial and incremental LMS processing for distributed estimation IEEE Transactions on Signal Processing, 2011, vol. 59, no. 4, p. 1465 to 1480. DOI: $10.1109 /$ TSP.2010.2100386

[26] GUI, G., XU, L., SHAN, L., et al. Adaptive MIMO channel estimation using sparse variable step-size NLMS algorithms. In IEEE International Conference on Communication Systems (ICCS). 2014, p. 605-609. DOI: 10.1109/ICCS.2014.7024874

[27] BERROU C., GLAVIEUX, A. Near optimum error correcting coding and decoding: Turbo-codes. IEEE Transactions on Communications, 1996, vol. 44, no. 10, p. 1261-1271. DOI: $10.1109 / 26.539767$ 
[28] TAKESHITA, O. Y., COLlinS, O. M., MASSEY, P. C., et al. A note on asymmetric turbo-codes. IEEE Communications Letters, 1999, vol. 3, no. 3, p. 69-71. DOI: 10.1109/4234.752905

[29] ZHAO, B., VALENTI, M. C. Distributed turbo coded diversity for relay channel. Electronics Letters, 2003, vol. 39, no. 10, p. 786 to 787. DOI: $10.1049 / \mathrm{el}: 20030526$

[30] ZHANG, Z., DUMAN, T. M. Capacity-approaching turbo coding and iterative decoding for relay channels. IEEE Transactions on Communications, 2005, vol. 53, no. 11, p. 1895-1905. DOI: 10.1109/TCOMM.2005.858654

[31] VUCETIC, B., YUAN, J. Turbo Codes: Principles and Applications, vol. 559: Springer US, 2012. ISBN: 978-1-46154469-2

[32] NEKUII, M., KISIALIOU, M., DAVIDSON, T. N., et al. Efficient soft-output demodulation of MIMO QPSK via semidefinite relaxation. IEEE Journal of Selected Topics in Signal Processing, 2011, vol. 5, no. $8, \quad$ p. 1426-1437. DOI: 10.1109/JSTSP.2011.2172772

[33] BENEDETTO, S., DIVSALAR, D., MONTORSI, G., et al. A softinput soft-output APP module for iterative decoding of concatenated codes. IEEE Communications Letters, 1997, vol. 1, no. 1 , p. 22-24. DOI: $10.1109 / 4234.552145$

\section{About the Authors ...}

Shoaib MUGHAL received his B.S. degree in Electronic Engineering from Sir Syed University of Engineering and Technology, Karachi, Pakistan, in 2008. In 2011, he obtained his M.E. degree in Telecommunication at NED University of Engineering and Technology, Karachi, Pakistan. He worked as a lecturer in the Department of Electronic Engineering at Sir Syed University of Engineering and Technology, Karachi, Pakistan, for three years. He is currently a Ph.D. research scholar at the College of Electronic and Information Engineering, Nanjing University of Aeronautics and Astronautics, China. His major research interests are multiple-input multiple-output systems, channel coding, coded-cooperative diversity and coded-modulation techniques.

Fengfan YANG received the M.Sc. and Ph.D. degrees from the Northwestern Polytechnical University and Southeast University, China in 1993, and 1997, respectively, all in Electronic Engineering. He has been with the College of Information Science and Technology, Nanjing University of Aeronautics and Astronautics since May 1997. From October 1999 to May 2003, he was a research associate at the Centre for Communication Systems Research, University of Surrey, UK, and Dept. of Electrical and Computer Engineering, McGill University, Canada. His major research interests are information theory, channel coding and their applications for mobile and satellite communications.

Saqib EJAZ received his B.Sc. (Hons.) degree in Electrical Engineering from the University of Engineering and Technology, Taxila, Pakistan, in 2006. Later, he did his M.S. Electronic Engineering from Ghulam Ishaq Khan Institute of Engineering Sciences and Technology (GIKI), KPK, Pakistan, in 2008. In 2015, he completed his Ph.D. degree from the College of Electronic and Information Engineering, Nanjing University of Aeronautics and Astronautics, China. He has more than 20 international journal/conference publications. His research interests include information theory, channel coding, multi-input multioutput systems, cooperative diversity, coded-modulation techniques, and spread spectrum systems.

Syed Hassan AHMED completed his B.S. degree in Electronic Engineering from Sir Syed University of Engineering and Technology, Karachi, Pakistan, in 2008. He received his M.S. degree in Wireless Communication at Brunel University, London, England, in 2009. He is currently pursuing his Ph.D. degree in the College of Electronic and Information Engineering, Nanjing University of Aeronautics and Astronautics, Nanjing, China. His research interests are channel coding and coded-cooperative communications

Rahim UMAR completed his B.Sc. degree in Electrical Engineering at the University of Engineering and Technology, Peshawar, Pakistan, in 2007. He worked as a lecturer in the Electrical Engineering Department at CECOS University Peshawar, Pakistan, for two years. In 2011, he completed his MS degree in Electrical Engineering, specializing in signal processing and wave propagation from Linnaeus University Växjö, Sweden. In late 2011 he joined Ghulam Ishaq Khan Institute of Engineering Science and Technology where he worked as Research Associate in the Department of Engineering Sciences. Presently, he is working as a Ph.D. research scholar in the field of channel coding at Nanjing University of Aeronautics and Astronautics, China. His research interests are signal processing and channel coding. 